\title{
||||||||||||||||||||||||||||||||||||||||||||||||||||||||||||||||||.
}

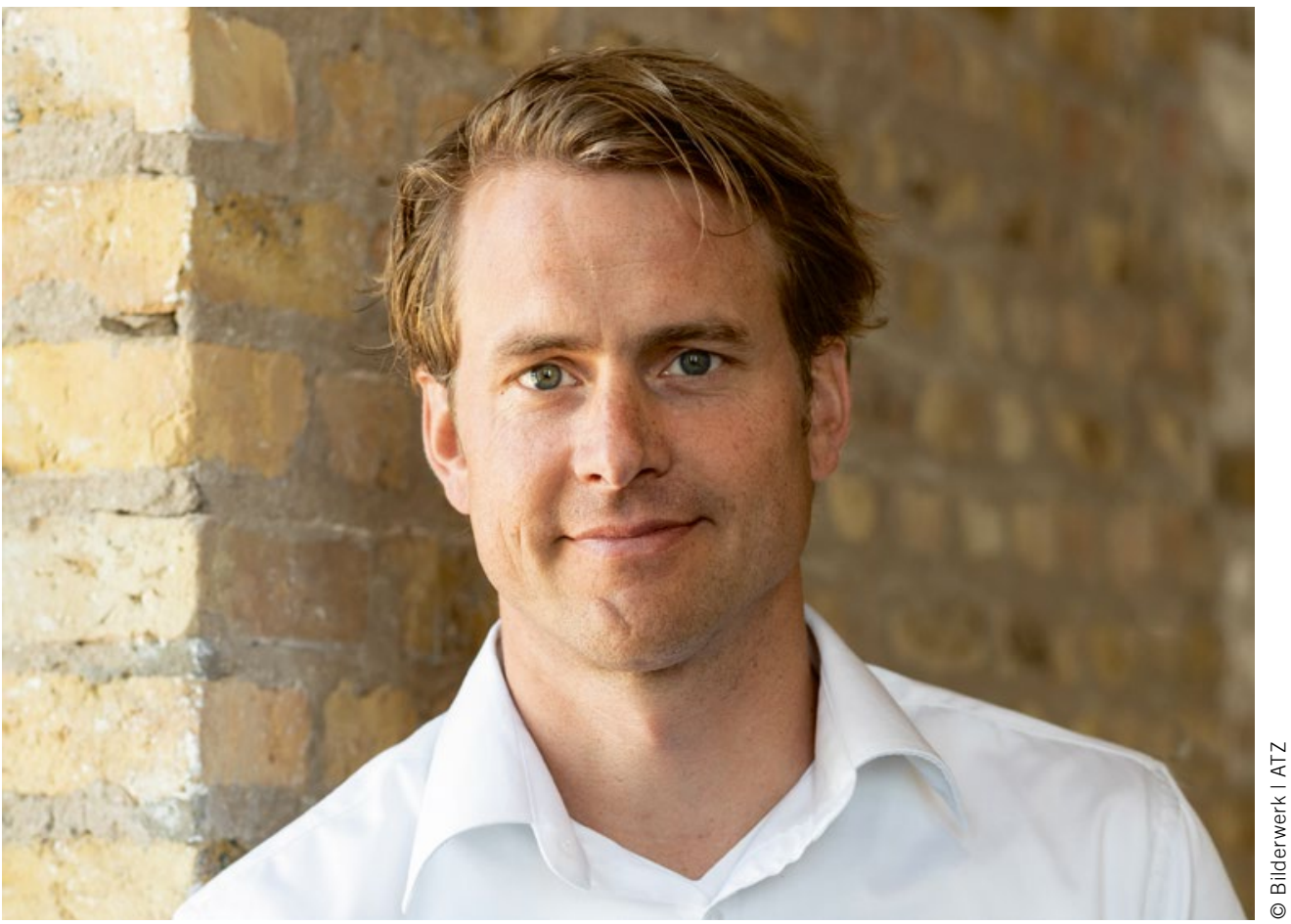

Dipl.-Ing. Jochen Tüting

Geschäftsführer der

Chery Europe $\mathrm{GmbH}$,

ATZ-Beirat

\section{Standards als Motor für Wirtschaftlichkeit}

Als ich 2013 mit meiner Familie nach China zog, entschieden wir uns für eine unmöblierte Wohnung, um uns selbst ein Zuhause für die nächsten Jahre zu schaffen. Auf der Suche nach europäischen Möbeln fanden wir uns schnell zwischen den Hochregallagern eines schwedischen Herstellers wieder. Tatsächlich sah der Kleiderschrank so aus wie in Deutschland, ließ sich genauso aufbauen, und selbst die auf Zeichnungen basierende Anleitung war identisch: ein einziges Produkt für den globalen Markt.

Was uns in diesem Kontext völlig normal vorkommt, ist in der Automobilindustrie die Ausnahme. OEMs und Zulieferer werden durch regionale Vorschriften zu einer massiven Komplexität in Entwicklung und Testing gezwungen. Ein Beispiel: Die Crashsicherheits-Anforderungen aus UN R137, GB 115512014 und FMVSS 208 verfolgen denselben Zweck: die Insassen bei einem Frontalzusammenstoß zu schützen. Allerdings werden in Europa kleinere Dummys auf der Beifahrerseite verwendet als in China und den USA. Dafür wird in den Staaten mit Tempo $56 \mathrm{~km} / \mathrm{h}$ getestet, bei uns in der EU und in China mit $50 \mathrm{~km} / \mathrm{h}$. Die Liste von noch deutlich gravierenderen Unterschieden wäre sehr lang.

Aufseiten der Hersteller bedeutet diese Heterogenität eine Multiplikation des Aufwands sowohl im Simulationsbereich als auch im Testing. In einigen Fällen reicht es zwar, den Worst Case abzusichern; spätestens bei der Homologation werden aber die physischen Tests gefordert. Es kann richtig sein, regionale Unterschiede zu berücksichtigen, wenn zum Beispiel mittlere Körpergrößen stark variieren. Die Tatsache, dass in Europa ein kleinerer Dummy auf der Beifahrerseite sitzt als in China, zeigt aber, dass dies derzeit nicht der Grund für die Differenzierung ist.

Die Globalisierung schreitet voran - die Corona-Krise zeigt uns dies überdeutlich auf. Es ist Zeit, dass die Anstrengungen im Bereich der Homogenisierung von Regularien endlich an Fahrt aufnehmen. Die Vereinten Nationen verfolgen gute Ansätze, und dennoch verschwendet die Automobilindustrie jedes Jahr Hunderte Millionen Euro für die Umsetzung unterschiedlicher gesetzlicher Anforderungen auf den regionalen Fahrzeugmärkten. Gewinner von globalen Standards wären alle: die Industrie durch weniger Komplexität, die Politik durch vereinfachte Genehmigungsverfahren und die Kunden, denen Pkw günstiger angeboten werden könnten.

Wir werden in Zukunft eine der größten Standardisierungen der letzten Jahrzehnte sehen - das Lenkrad wird irgendwann verschwunden sein. Keine Links-Rechts-Varianten mehr für Instrumententafel, Lenkgetriebe, Bremsleitungen etc. Die Einsparungen werden enorm sein.

Wenn wir uns Pkw ohne Lenkrad vorstellen können, dann sollten doch einheitliche Emissions- oder Sicherheitsregularien auch möglich sein. 